
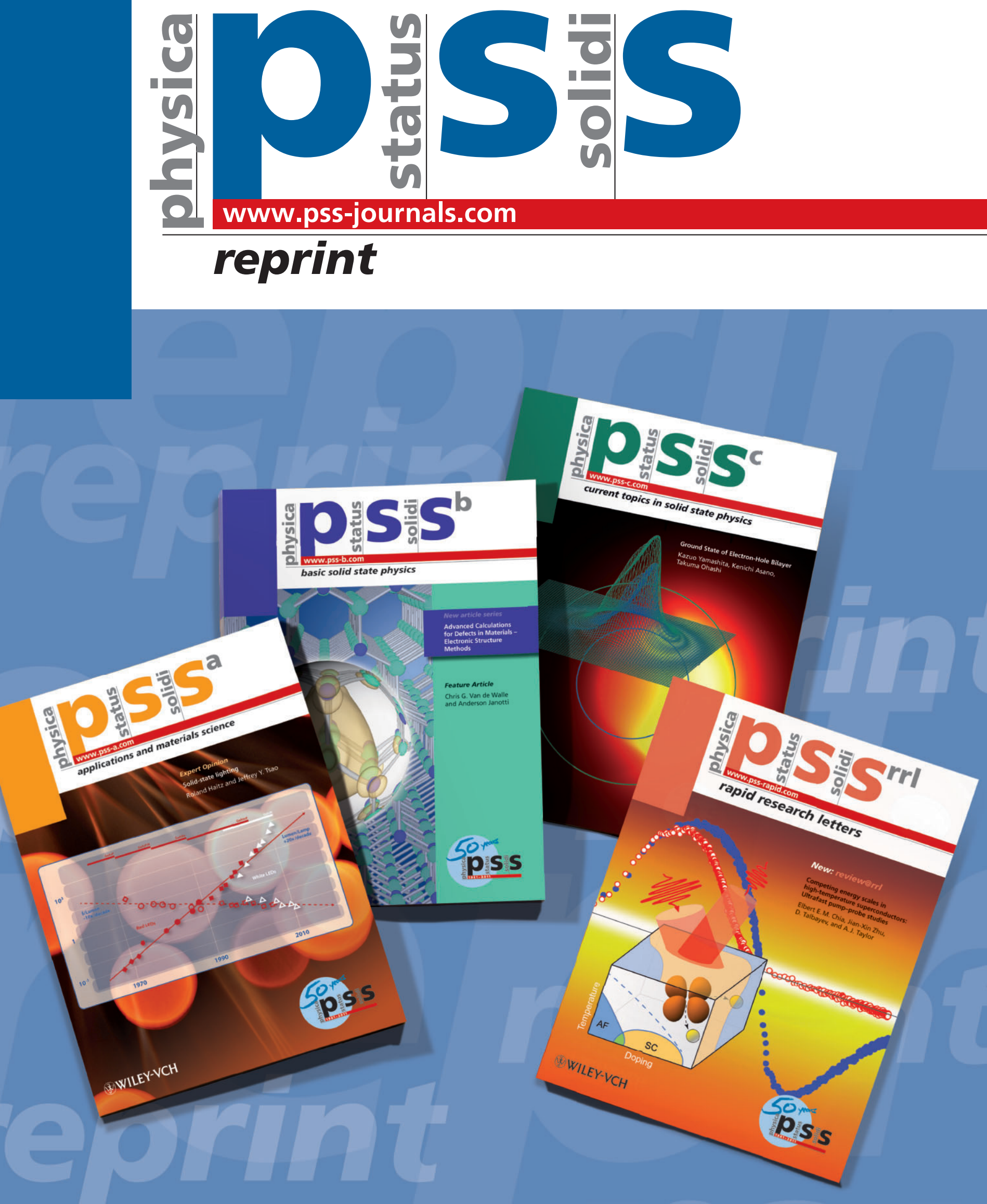


\title{
Nanocontact size dependence of the properties of vortex-based spin torque oscillators
}

\author{
R. M. Otxoa ${ }^{*, 1,2}$, M. Manfrini ${ }^{3,4}$, T. Devolder ${ }^{1,2}$, J.-V. Kim ${ }^{1,2}$, W. van Roy ${ }^{3}$, L. Lagae ${ }^{3,4}$, and C. Chappert ${ }^{1,2}$ \\ ${ }^{1}$ Institut dElectronique Fondamentale, UMR CNRS 8622, 91405 Orsay cedex, France \\ ${ }^{2}$ Universite Paris-Sud, UMR 8622, 91405 Orsay, France \\ ${ }^{3}$ IMEC, Kapeldreef 75, 3001 Leuven, Belgium \\ ${ }^{4}$ Laboratorium voor Vaste-Stoffysica en Magnetisme, K. U. Leuven, Celestijnenlaan 200 D, 3001 Leuven, Belgium
}

Received 18 October 2011, revised 2 March 2011, accepted 2 March 2011

Published online 21 June 2011

Keywords magnetic vortex, nanocontacts, oscillations

*Corresponding author: e-mail ruben.otxoa@u-psud.fr, Phone: (+33) 1691540 13, Fax : (+33) 169157841

We study the frequency, linewidth, and power of spin torque driven vortex oscillators, based on a nanocontacted spin-valve (SV). The oscillation frequency strongly decreases with the contact size, and increases with the current. The power delivered by the oscillator is not quadratic with the current, in contrast with the behavior expected from the rigid vortex model (RVM). The linewidth is almost independent of the current at low current and does not strongly depend on the nanocontact size. We compare our findings with the outcomes of the RVM.
1 Introduction Magnetic vortices have recently received much attention [1-3] in the field of micromagnetism since it was discovered that they are fundamental states on the microscopic and nanoscopic scale which have potential applications in information storage and spintronic devices. The spin transfer phenomenon [1], where a polarized current transfers angular momentum to the magnetic moments of a ferromagnetic material provides a new method to manipulate the magnetization without the application of any external field. In the system that we present here instead of taking advantage of the confined magnetic structures we use a typical magnetic system called spin-valve (SV) with a metallic nanocontact on the top of this magnetoresistive structure. The current is injected into the magnetic multilayer which are normally composed by ferromagnetic layers separated by a nonmagnetic layer. In this kind of system, large current densities flow through the magnetic multilayer structure which can lead to large spin torque effects. Under certain conditions the Oersted-Ampere field associated with the current can nucleate a vortex [4] which will be carried into a steady state of motion around the point contact (PC) $[2,5]$. These self-sustained oscillations are generated in the 50$500 \mathrm{MHz}$ range.
It has been experimentally demonstrated recently that vortex oscillators have characters that make them very interesting for potential applications such as its high quality factor, agility [6], and that the gyrotropic motion is controllable without the application of additional large magnetic fields $[4,7]$.

In this paper, we present an experimental study of the PC size dependence of the oscillation frequency in a magnetic nanocontact system. Also other characteristic features like linewidth and power will be discussed. Notice that these experiments were made under zero in plane and perpendicular fields, which means there is no vortex in the initial state. In the last section we will compare the latest theories presented to date and our experimental results.

2 Samples and experimental setup The experimental system studied is a metallic nano-PC fabricated on top of our SV. We use nanocontacts with nominal radii of 40 , $50,60,80 \mathrm{~nm}$ most of them showing vortex oscillations. The nominal size can be quite different from the physical or "real" size of the PC, therefore this will be discussed in Section 3. The composition of the SV is the following: $\mathrm{Ni}_{80} \mathrm{Fe}_{20}(3) \operatorname{IrMn}(6) / \mathrm{Co}_{90} \mathrm{Fe}_{10}(4.5) / \mathrm{Cu}(3.5) / \mathrm{Ni}_{80} \mathrm{Fe}_{20}(4) / \mathrm{Pt}(3)$. The number in parentheses denote the layer thicknesses in nanometers. In this structure (see Fig. 1) the layer which 

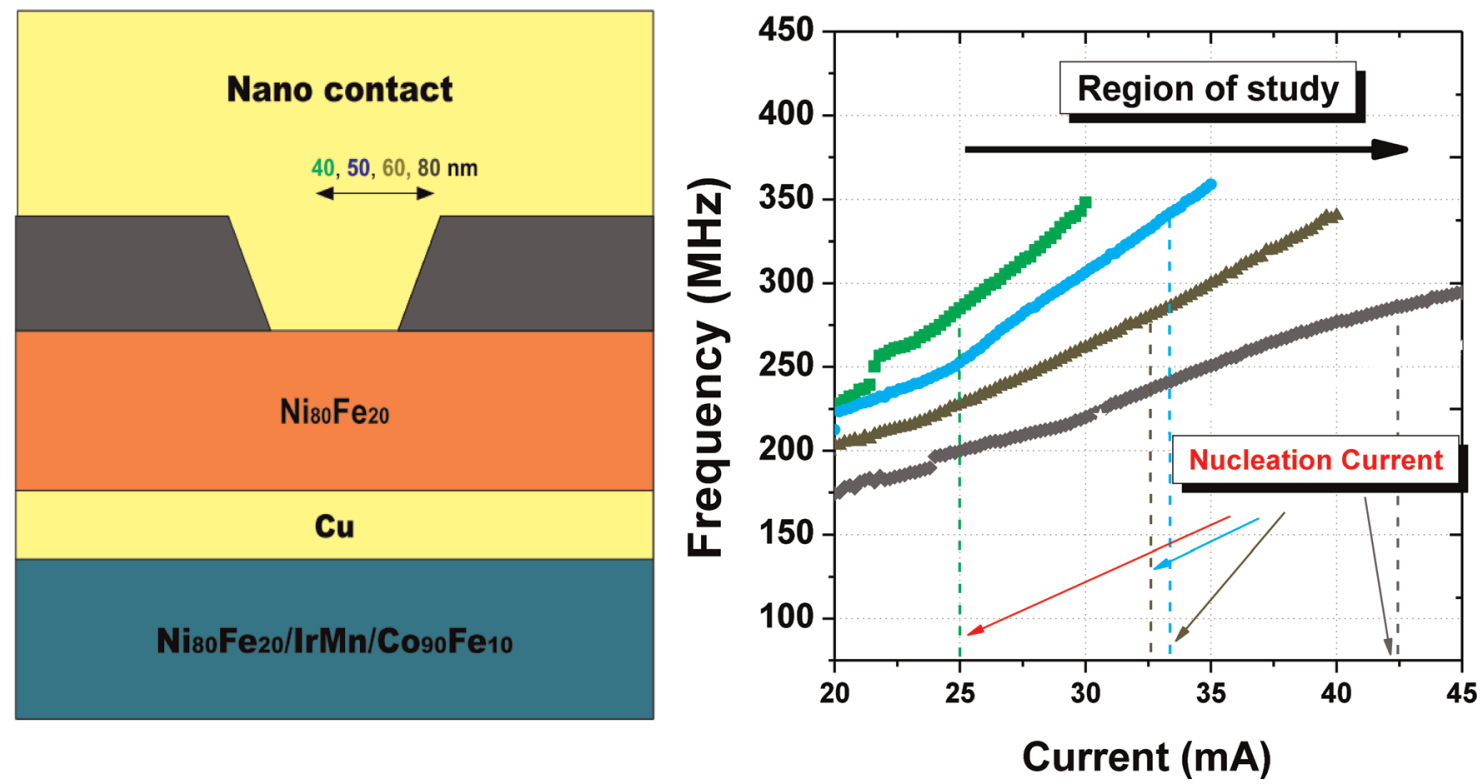

Figure 1 (online colour at: www.pss-b.com) Frequency versus current for different nano-contact radius of 40 (top green curve), 50, 60, 80 (bottom gray curve) $\mathrm{nm}$. Dots represent experimental data. Throughout all of this paper we consider the same code of colors for each PC size.

plays the role of the free layer (FL) is $\mathrm{Ni}_{80} \mathrm{Fe}_{20}(4)$ while $\mathrm{CoFe}(4.5)$ layer is exchange biased by the $\operatorname{IrMn}(6)$, an antiferromagnet that serves as a pinned layer (PL) for the giant magnetoresistance (GMR) variations. Details in fabrication process have been given elsewhere [5].

The device was measured at room temperature and the magnetization dynamics was detected by electrical measurements via the GMR effect. The experimental procedure to study the dependence between oscillation frequency and the applied current have been described elsewhere [5].

\section{Results}

3.1 Nucleation current versus PC size Starting from zero current, it is necessary to apply a threshold current amplitude to nucleate the vortex [5] (see Fig. 1). The threshold current usually called nucleation current $I_{\text {nucl }}$ increases with the PC size, being $25 \mathrm{~mA}$ for smallest $\mathrm{PC}$ and $42 \mathrm{~mA}$ for the biggest one (see Fig. 1). It is possible to maintain this self-sustained oscillations till values in the order of $6-10 \mathrm{~mA}$ [4]. Once the vortex is nucleated, the frequency depends quasi-linearly with the current (see Fig. 1). We focus on this regime, where the vortex shows a dynamical behavior.

3.2 Estimate of real PC size The aim now is to extract the physical size of the PC and find a general trend between frequency and "real" PC. To do that we used two different methods. In the first one, we take into account that the total circuit resistance is the sum of the resistance of the electrodes $R_{\text {elec }}$ plus a contribution due to the relative magnetic orientation between the PL and FL which we call $\Delta R$; GMR represents the GMR ratio which is extracted from the fit [see Eq. (1)]:

$$
R_{\mathrm{t}}=R_{\text {elec }}+\frac{\Delta R}{\mathrm{GMR}} .
$$

In the second method, we use the fact that it is possible to find a relation between the nucleation current and the total resistance of our device:

$$
R_{\mathrm{t}}=R_{\text {elec }}+\frac{K}{I_{\text {nucl }}} .
$$

In the first method, depending on the size of the PC we obtain different values for the total resistance $R_{\mathrm{t}}$ and $\Delta R$ which allow us to get from the slope $R_{\mathrm{t}}$ versus $\Delta R$ the resistance of the electrodes, which is independent on the size of the PC. Typically the values for $\Delta R$ are $25 \mathrm{~m} \Omega$ for smallest and $17 \mathrm{~m} \Omega$ for the biggest PC. The value for $R_{\text {elec }}$ is $5.8 \pm 0.3 \Omega$. The value of GMR extracted is $1.1 \%$. Once this is done we can directly obtain the resistance of the PC. $R_{\mathrm{pc}}=R_{\mathrm{t}}-R_{\text {elec }}=1.2-2.3 \Omega$. In the second method we use the same procedure, extract the resistance of PC from the fit. Considering the above assumptions together with the fact that the resistance of the $\mathrm{PC}$ is directly proportional to the inverse of the square of the PC radius, $R_{\mathrm{pc}}=K / a_{\mathrm{pc}}^{2}$, it is possible to extract the real values of the different PC sizes. To do that, we do the strong assumption that the nominal radius of the PC of $100 \mathrm{~nm}$ coincides with its real radius size. This allows us to directly deduce the physical radius of each of the other points of contact and be able to get as close as possible to real relation between the slope and radius of the contact point. In Fig. 2 is represented slope versus $a_{\mathrm{pc}}^{-2}$ taking into account the real PC size. We must say that as can be seen in 

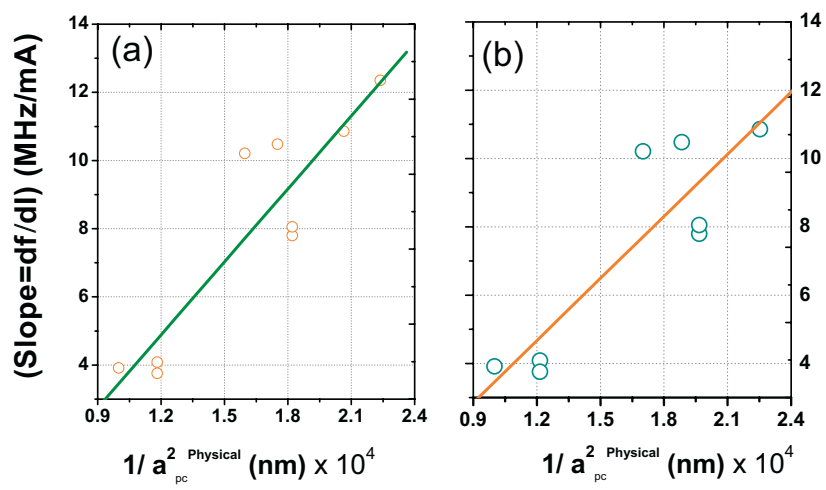

Figure 2 (online colour at: www.pss-b.com) Representation of slope versus $a_{\mathrm{pc}}^{-2}$ by two different methods. (a) is obtained using $\Delta R$ and (b) is obtained using $1 / I_{\text {nucl }}$.

both Fig. 2(a) and (b) the real PC's sizes do not change too much for each method used, which means that the assumption about how to extract the physical sizes was consistent between both. The fabricated devices have a physical size slightly bigger than intended, with a radius size offset of $15 \mathrm{~nm}$. We find a relation between oscillation frequency of the vortex around the $\mathrm{PC}$ and the $\mathrm{PC}$ radius:

$$
f \propto \frac{1}{a_{\mathrm{pc}}^{2}}
$$

3.3 Frequency versus PC size In Fig. 3, we study the slope $(\mathrm{d} f / \mathrm{d} I)$ versus the nominal PC size, we see a strong decreasing in the slope versus the PC size, which means that the PC size plays an important role in the oscillation frequency of the vortex.

3.4 Power dependence with PC size The power delivered to the measurement circuit comes from the vortex orbital motion in the $\mathrm{PC}$ region. The vortex motion gives rise a time-varying change in the magnetoresistance $\left(\Delta R_{\mathrm{dyn}}\right)$. It is possible to estimate the radius of the vortex orbit during its

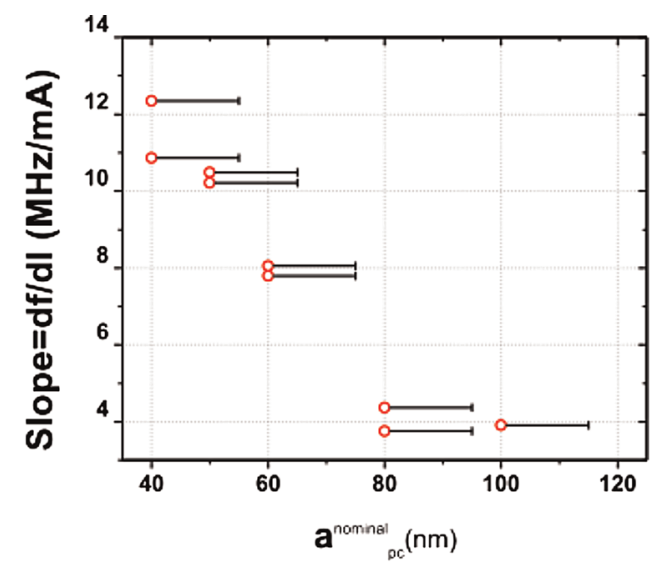

Figure 3 (online colour at: www.pss-b.com) Dependence of frequency versus current slope with the nanocontact size. motion. The procedure is the following. If the sample resistance varies like $\left(\Delta R_{\mathrm{dyn}} / 2\right) \cdot \sin (\omega t)$ and the device is submitted to an $I_{\mathrm{dc}}$ current while connected in series with $Z_{\text {load }}$ at its both electrodes, it will deliver to the measurement circuit the following power:

$$
P=\frac{Z_{\text {load }} \cdot \Delta R_{\mathrm{dyn}}^{2} \cdot I_{\mathrm{dc}}^{2}}{8 \cdot\left(Z_{\text {load }}+Z_{\text {out }}\right)^{2}},
$$

where $Z_{\text {load }}=50 \Omega$ and $Z_{\text {out }}=Z_{\text {load }}+R_{\text {dut }}=55.8 \Omega$ which is the sum of $50 \Omega$ series resistance and the resistance of the device including its electrodes. Considering the "Rigid vortex model" (RVM) [8] in which it is assumed that the vortex can be considered as a rigid object (even when it is moving) without internal degrees of freedom, it is possible to express analytically the value of $\Delta R_{\mathrm{dyn}}$ in terms of the radius of the orbit that the vortex follows, simply solving the integral of Eq. (5):

$$
\begin{aligned}
\Delta R_{\mathrm{dyn}}\left(\boldsymbol{r}_{\mathrm{vx}}\right) & =\frac{R_{\mathrm{pc}} \cdot \mathrm{GMR}}{2} \iint_{\mathrm{PC}} \boldsymbol{m}(\boldsymbol{r})_{\mathrm{fl}} \cdot \boldsymbol{m}(\boldsymbol{r})_{\mathrm{fx}} \cdot \mathrm{d} \boldsymbol{r} \\
& =\frac{R_{\mathrm{pc}} \cdot \mathrm{GMR}}{2} \iint_{\mathrm{PC}} \frac{(r-R \cdot \cos (\phi)) \mathrm{d} r \mathrm{~d} \phi}{\sqrt{R^{2}+r^{2}-2 \cdot r \cdot R \cdot \cos (\phi)}} .
\end{aligned}
$$

To simplify the calculations, it is assumed that the magnetization of the PL remains static and plays no role in the dynamics of the vortex which relies in the FL. $\boldsymbol{m}(\boldsymbol{r})_{\mathrm{fl}}$ and $\boldsymbol{m}(\boldsymbol{r})_{\mathrm{fx}}$ refer to magnetization of the FL and fixed layer respectively. $\boldsymbol{r}$ and $\boldsymbol{R}$ represent position vectors in polar coordinates of the point of study and the vortex core position, respectively.

In Fig. $4, R_{\text {orbit }} / a_{\mathrm{pc}}$ represents the ratio between the orbit radius of the vortex and the $\mathrm{PC}$ radius. It is easy to see that when the vortex is far away from the PC we get $\Delta R_{\mathrm{dyn}}=R_{\mathrm{pc}} \cdot \mathrm{GMR}$. This would mean that the vortex has been expelled and now our magnetic layers relies uniform magnetized (without vortex).

From the power versus current for different PC sizes we see an interesting trend for each PC size. After a value of current we can see an important decreases in the power. This is something unexpected but can be explained by at least two different scenarios. First one, contrary with the RVM [8] predicts, the orbit radius could be dependent with current. In this scenario the decay process of the orbit trajectory of the vortex behind the PC region could give rise a decreases in $\Delta R_{\mathrm{dyn}}$ as can be seen in Fig. 4. One way to check this possible explanation is to apply perpendicular field and see whether the $\Delta R_{\text {dyn }}$ changes or not. As we know the confining potential created by the current would be reduced by this perpendicular fields, which would lead to a variation in the orbit of the vortex.

Another explanation for the decrease in the power could come from the fact that the PL is not completely pinned and is distorted by the presence of the current. This would make a competition between the uniform field from the layer and the Oersted field generated by the current. This could be tested 

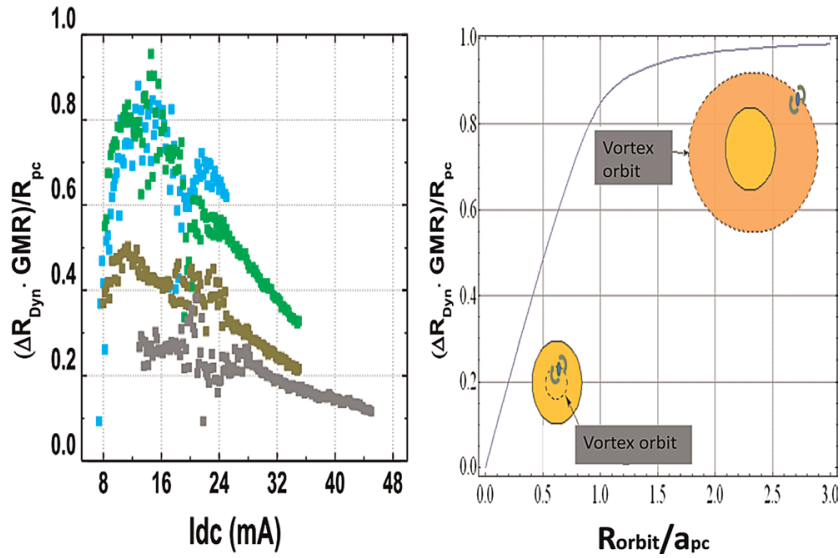

Figure 4 (online colour at: www.pss-b.com) Figure on the left represents experimental data of $\Delta R_{\mathrm{dyn}}=R_{\mathrm{pc}}$. GMR versus $I_{\mathrm{dc}}$ and right figure represents numerical solution of the Eq. (5) for $\Delta R_{\mathrm{dyn}}=R_{\mathrm{pc}} \cdot \mathrm{GMR}$ as function of the position of the vortex in the layer in which it relies, taking the reference system in the center of the PC.

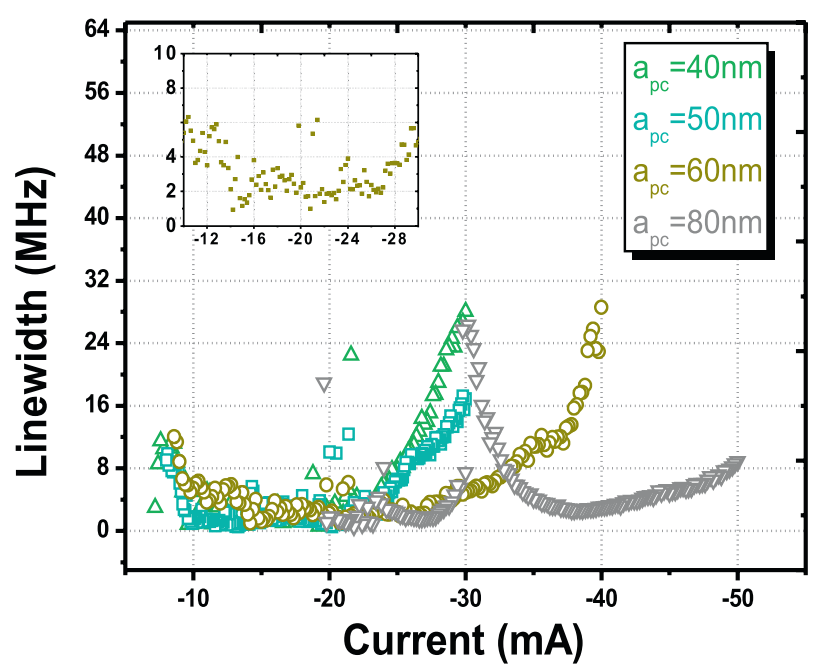

Figure 5 (online colour at: www.pss-b.com) Linewidth versus current for different PC sizes. Inset figure represents the linewidth versus current for the PC $a_{\mathrm{pc}}^{\text {nominal }}=60 \mathrm{~nm}$.

by applying small fields along the in-plane direction watching whether there is a variation again in the power spectra.

3.5 Linewidth versus current Another important feature of the vortex motion is related to the linewidth. As can be seen in Fig. 5, very low linewidths are reached for every PC when we are in the range of 10-25 mA. As can be seen in the inset figure of Fig. 5, the lowest linewidth reached is $1.2 \mathrm{MHz}$ which corresponds to a PC size of $60 \mathrm{~nm}$ nominal radius. In the RVM $\Delta f$ is directly proportional to the inverse of the square of the orbit radius:

$$
\Delta f \propto \frac{1}{R_{0}^{2}} .
$$

We show in Fig. 5 that when the current reaches $25 \mathrm{~mA}$, suddenly the linewidth increases, this means (following the RVM approach) that the orbit of the vortex has decreased substantially. It is possible to see in Fig. 4 that also there is a transition regime around (24-25) $\mathrm{mA}$ from which $\Delta R_{\text {dyn }}$ suffers an important decay. This would give more strength on the idea of the reduction of the radius trajectory orbit of the vortex when we increase enough the current.

4 Discussion and concluding remarks Let us summarize our experimental results, and compare them to the prediction of the RVM. Nucleation current is consistent with a current density-based mechanism. Frequency slope decreases with PC size, in contrast to predictions done from the RVM that conclude $\mathrm{d} f / \mathrm{d} a_{\mathrm{pc}}=0$. Power versus current curve is consistent with the vortex orbit $R_{0}$, being the most extended near $12-16 \mathrm{~mA}$. This corresponds to a shallow linewidth minimum, consistent with the predictions that $\Delta f$ decreases with $R_{0}$. For 40 and $50 \mathrm{~nm}$ we can certify that at low currents 10-20 mA the vortex orbits outside of the PC. At large current, there is a systematic drastic increase of linewidth whose origin is unclear. Our results call for a more elaborate theory of vortex based dynamics taking into account the real geometry.

Acknowledgements The authors would like to thank Sebastien Petit for his suggestions about key question of this work. This work has been supported by the European community under 7th FP for the Marie Curie ITN SEMISPINNET.

\section{References}

[1] D. V. Berkov and N. Gorn, L Phys. Rev. B 80, 064409 (2009).

[2] M. R. Pufall, W. H. Rippard, M. L. Schneider, and S. E. Russek, Phys. Rev. B 75, 140404 (2007).

[3] R. Hertel, S. Gliga, M. Fahnle, and C. M. Schneider, Phys. Rev. B 98(11), 117201 (2007).

[4] T. Devolder, K. Joo-Von, P. Crozat, C. Chappert, M. Manfrini, M. van Kampen, W. Van Roy, L. Lagae, G. Hrkac, and T. Schrefl, Appl. Phys. Lett. 95, 012507 (2009).

[5] Q. Mistral, M. van Kampen, G. Hrkac, J.-V. Kim, T. Devolder, P. Crozat, C. Chappert, L. Lagae, and T. Schrefl, Phys. Rev. Lett. 100, 25701 (2008).

[6] M. Manfrini, T. Devolder, J.-V. Kim, P. Crozat, C. Chappert, M. van Kampen, W. Van Roy, L. Lagae, G. Hrkac, and T. Schrefl, Appl. Phys. Lett. 95, 192507 (2009).

[7] M. van Kampen, L. Lagae, G. Hrkac, T. Schrefl, J.-V. Kim, T. Devolder, and C. Chappert, J. Appl. Phys. 42, 245001 (2009)

[8] K. Joo-Von and T. Devolder, arXiv: 1007 3859v1, July 2010. 